\title{
On the forces that drive and resist deformation of the south-central Mediterranean: a mechanical model study
}

\author{
Nicolai Nijholt, Rob Govers, and Rinus Wortel \\ Utrecht University, Faculty of Geosciences, Earth Sciences, Utrecht, Netherlands (n.nijholt@uu.nl)
}

The Mediterranean region displayed transient behavior throughout the last 30 Myrs, with ephemeral fault activity and strong variation in deformation intensity. This suggests that the tectonic forces changed rapidly as well, both in time and space. The main forces are associated with the large-scale Miocene to Quaternary processes: 1) slow Africa-Eurasia plate convergence ( $\sim 5 \mathrm{~mm} / \mathrm{yr})$, and 2 ) slab rollback, with the trench and the Calabrian block moving mainly towards the SE, resulting in a migrating Aeolian volcanic arc and opening of the Tyrrhenian back-arc basin.

Today, these forces control active tectonics where the observations also display a strong variation in deformation style. We aim to understand the current kinematics in southern Italy and Sicily in terms of lithospheric forces that cause them. The strong regional variation of geodetic velocities appears to prohibit such simple explanation. We use mechanical models to quantify the present-day deformation resulting from large-scale Africa-Eurasia convergence, ESE retreat of the Calabrian subduction zone, pull by the Aegean slab, and regional variations in gravitational potential energy (topography). A key model element is the friction on major regional fault zones. We show that geodetic velocities, seismicity and sense of slip on regional faults can be understood to result from lithospheric forces.

Our most important model result is that regional variations in fault friction are required to fit the observations, notably very low friction on the Calabrian subduction contact, and a buildup towards a significant earthquake on the Cittanova fault. We also find that the Calabrian net slab pull force is strongly reduced (compared to the value possible in view of the slab's dimensions). Such a very small contribution of the Calabrian net slab pull to the present-day force balance in the south-central Mediterranean suggests that the Calabrian arc is now further transitioning towards a setting dominated by Africa-Eurasia plate convergence, whereas during the past 30 Myrs slab retreat continually was the dominant factor. 